\title{
Toward an Energy Transfer and Interacting Scale Analysis of MHD Turbulence with Implications for Space and Astrophysical Plasmas
}

\author{
Y. Zhou, O. Schilling, S. Ghosh
}

U.S. Department of Energy

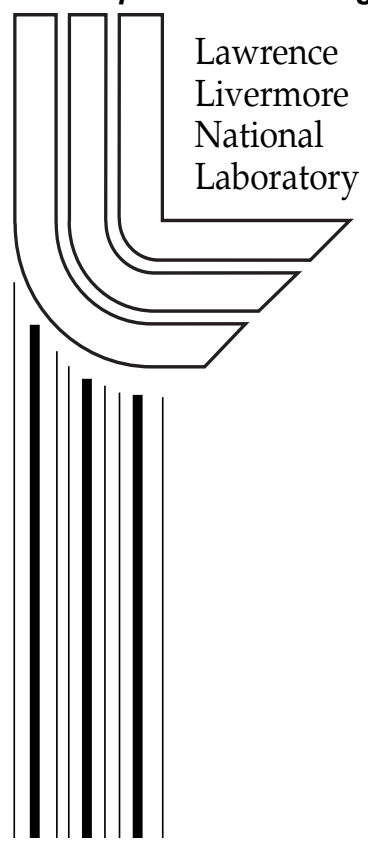

This article was submitted to

$32^{\text {nd }}$ American Institute of Aeronautics and Astronautics

Plasmadynamics and Lasers Conference, Anaheim, CA, June 1114,2001

\section{April 11, 2001}




\section{DISCLAIMER}

This document was prepared as an account of work sponsored by an agency of the United States Government. Neither the United States Government nor the University of California nor any of their employees, makes any warranty, express or implied, or assumes any legal liability or responsibility for the accuracy, completeness, or usefulness of any information, apparatus, product, or process disclosed, or represents that its use would not infringe privately owned rights. Reference herein to any specific commercial product, process, or service by trade name, trademark, manufacturer, or otherwise, does not necessarily constitute or imply its endorsement, recommendation, or favoring by the United States Government or the University of California. The views and opinions of authors expressed herein do not necessarily state or reflect those of the United States Government or the University of California, and shall not be used for advertising or product endorsement purposes.

This is a preprint of a paper intended for publication in a journal or proceedings. Since changes may be made before publication, this preprint is made available with the understanding that it will not be cited or reproduced without the permission of the author.

This work was performed under the auspices of the United States Department of Energy by the University of California, Lawrence Livermore National Laboratory under contract No. W-7405-Eng-48.

This report has been reproduced directly from the best available copy.

Available electronically at http://www.doc.gov/bridge

Available for a processing fee to U.S. Department of Energy

And its contractors in paper from

U.S. Department of Energy

Office of Scientific and Technical Information

P.O. Box 62

Oak Ridge, TN 37831-0062

Telephone: (865) 576-8401

Facsimile: (865) 576-5728

E-mail: reports@adonis.osti.gov

Available for the sale to the public from

U.S. Department of Commerce

National Technical Information Service

5285 Port Royal Road

Springfield, VA 22161

Telephone: (800) 553-6847

Facsimile: (703) 605-6900

E-mail: orders@ntis.fedworld.gov

Online ordering: http://www.ntis.gov/ordering.htm

OR

Lawrence Livermore National Laboratory

Technical Information Department's Digital Library

http://www.llnl.gov/tid/Library.html 


\title{
TOWARD AN ENERGY TRANSFER AND INTERACTING SCALE ANALYSIS OF MHD TURBULENCE WITH IMPLICATIONS FOR SPACE AND AST ROPHYSICAL PLASM AS ${ }^{\infty}$
}

\author{
Ye Zhou and Oleg Schilling \\ University of $\mathrm{C}$ alifornia, Lawrence Livermore National Laboratory \\ Livermore, CA 94551 \\ Sanjoy Ghosh \\ E mergent Information Technologies Inc. \\ Largo, M D 20774
}

\begin{abstract}
MHD turbulence has numerous applications in space and astrophysical plasmas. In this paper, the eddy-damped quasi-normal Markovian (EDQNM) model is used to perform a preliminary study of the nonlinear transfer process in three-dimensional MHD turbulence. B oth two- and three-dimensional contour plots of the triadic transfer functions are presented for the case of assumed energy spectra corresponding to K olmogorov inertial subrange scaling.

\section{Introduction}

The magnetohydrodynamic (MHD) approximation has been quite successful in space physics and astrophysics. In particular, the manifestation of turbulence and other nonlinear phenomena in astrophysical plasmas is explainable from an MHD turbulence perspective. The MHD description has been shown to be an excellent starting point for describing plasma motions when the macroscopic level of motions are well separated from the Coulomb collision/ particle gyro-scales.
\end{abstract}

${ }^{x}$ Copyright ${ }^{\circ} \mathrm{C} 2001$ by the American Institute of Aeronautics and Astronautics, Inc. All rights reserved.
The application of MHD turbulence to the solar wind illustrates how the understanding of basic plasma physics and the universe can be improved. The existence of the solar wind was deduced in 1896 by B irkeland and later theoretically predicted by Parker. Subsequent observations con ${ }^{-}$rmed the presence of hot, supersonic out ${ }^{\circ}$ ows of electrons, protons, and alpha particles from the upper limits of the corona of the Sun. The solar wind streams past the magnetosphere of the Earth, and is the means by which mechanical energy is transmitted from the Sun to the Earth. Solar wind spacecraft observations provide a readily available 'laboratory' for testing theories and assumptions. For example, spacecraft observations demonstrated that the solar wind can be characterized as a turbulent magnet $0^{\circ}$ uid. Reduced power spectra constructed from Mariner 10 spacecraft magnetometer data revealed a steady power-law spectrum spanning nearly three decades in frequency, with an ! i $5=3$ powerlaw, where ! is the spacecraft rest frequency. Relating time measurements to spatial scales using the Taylor frozen-in- ${ }^{\circ}$ ow hypothesis, this translates to a $k^{i} 5=3$ wavenumber spectrum, reminiscent of the well-known kinetic energy spectrum in fullydeveloped homogeneous, isotropic ${ }^{\circ}$ uid turbulence. 
Fyfe, $M$ ontgomery, and J oyce ${ }^{1}$ argued that the original Kolmogorov scaling, and its associated $\mathrm{K}^{\mathrm{i}} 5=3$ power-law, is also applicable to MHD turbulence. $\mathrm{K}$ raichnan ${ }^{2}$, however, proposed that the usual phenomenological argument should be modi ${ }^{-}$ed to include magnetic - eld e ${ }^{\circledR e c t s}$, which leads to a $\mathrm{k}^{\mathrm{i}} 3=2$ spectrum. Unquestionably, spacecraft observations have strongly motivated the study of turbulence in MHD models describing the dynamics of the solar wind.

Much MHD turbulence research has focused on the spectra of three quadratic, integral \rugged' invariants ${ }^{3 ; 4}$. These invariants are deduced from the incompressible, non-dissipative ap proximation of the MHD equations in the absence of a mean magnetic - eld. In three-dimensional MHD turbulence, these invariants are the energy (per unit mass), the cross helicity, and the magnetic helicity. Some interesting applications of M HD turbulence to the solar wind include the evolution of cross helicity, the devel opment of anisotropies, the decay of magnetic helicity with a mean magnetic - eld, and nearly-incompressible dynamics.

W hile signi ${ }^{-}$cant progress has been made, some fundamental aspects of MHD turbulence must be investigated and understood regarding the energy transfer and interacting scales. One can appreciate this point by noting that nearly all MHD turbulence, including its applications to the solar wind ${ }^{5\{7}$ rely on assumptions regarding the energy transfer process through the inertial range. Spacecraft observations may be able to indicate the total energy at a given scale in the spectrum, and detailed information on the energy transfer and interacting scales can be obtained by an analysis similar to that carried out for ${ }^{\circ}$ uid turbulence ${ }^{8\{10}$.

In this paper, we will develop the basic concepts and equations for the energy transfer and interacting scale analysis. After forming the transfer spectra, we will formulate the principal quantitative measurements for describing the spectral locality, strength, and anisotropies of the nonlinear modal couplings. All of these analyses can be carried out with direct numerical simulation (DNS) databases of MHD turbulence. The main limitation of DNS data is that the ${ }^{\circ}$ uid and magnetic $R$ eynolds numbers are restricted to relatively moderate values. Here, we present an alternative method for performing the energy transfer analysis using transfer spectra constructed from the eddydamped quasi-normal Markovian (ED QN M) closure model $^{4 ; 11}$. The EDQNM closure can achieve very high R eynolds numbers, and therefore, a wide range of spectral scales for the analysis.

\section{The MHD Equations}

The standard, unforced MHD equations are the $\mathrm{N}$ avier-Stokes equation

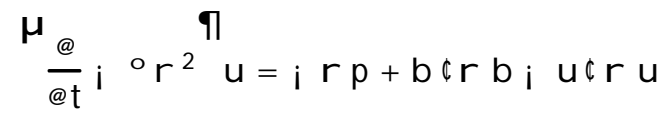

and the magnetic "eld equation

$$
\frac{\mu}{\text { @ }} i{ }^{3} r^{2} b=r f(u f b)
$$

with

$$
r \quad \phi u=r \quad \phi b=0 ;
$$

where the kinematic viscosity and magnetic di ßusivity are 0 and ${ }^{3}$, respectively.

F or homogeneous turbulence, the MHD equations in wavenumber space are

$$
\begin{gathered}
=M_{\phi} M_{i j k}(k)\left[u_{j}(p ; t) u_{k}(q ; t) ; b(p ; t) b_{k}(q ; t)\right] \\
={ }^{\mu} M_{i j k}^{\infty}(k) b_{j}(p ; t) u_{k}(q ; t) ;
\end{gathered}
$$

where the symbol $\phi$ in the sum represents the summation over wavevectors $p$ and $q$ with the triadic restriction $\mathrm{k}=\mathrm{p}+\mathrm{q}$. Here the symbol $\mathrm{z}_{\mathrm{ijk}}$ is the unit antisymmetric tensor and

$$
\begin{gathered}
M_{i j k}(k)=i \frac{i}{2}\left[k_{k} P_{i j}(k)+k_{i} P_{i k}(k)\right] ; \\
M_{i j k}^{B}(k)=i^{2}{ }_{i l m} k_{l^{2}}{ }_{m j k} ;
\end{gathered}
$$

where

$$
P_{i j}(k)= \pm_{j} i \frac{k_{i} k_{j}}{k^{2}}
$$

is the solenoidal projection tensor. 


\section{The Transfer Spectra}

The kinetic energy spectrum evolution equations can be constructed from the wavenumber space MHD equations. For example, the total energy spectrum evolution equation

$$
\frac{@}{\text { @ }}+2\left(\underline{o}+{ }^{3}\right) k^{2} E(k ; t)={ }_{\Phi}^{X} T_{E}(k ; p ; q) ;
$$

where the total energy spectrum is

$$
\begin{gathered}
E(k ; t) \\
=\frac{1}{2} h u_{i}(k ; t) u_{i}(k ; t)^{a}+b(k ; t) b(k ; t)^{a} i
\end{gathered}
$$

and the triadic interaction function of energy is de- ned as

$$
\begin{gathered}
T_{E}(k ; p ; q)=\stackrel{i}{ } M_{i j k}(k) u_{i}(k ; t)^{a} \\
f\left[u_{j}(p ; t) u_{k}(q ; t) ; b(p ; t) b_{k}(q ; t)\right] \grave{A} \\
+M_{i j k}^{B}(k) b(k ; t)^{a} b(p ; t) u_{k}(q ; t)+c: C:
\end{gathered}
$$

Similarly, taking the magnetic Prandt I number

$$
\begin{gathered}
\operatorname{Pr}_{m}=\frac{\varrho}{3} \\
=1 ;
\end{gathered}
$$

the cross helicity spectrum evolution equation is

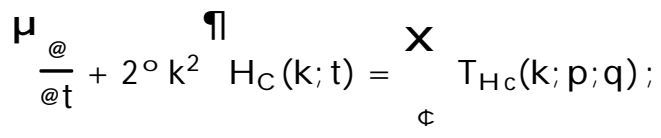

where the cross helicity spectrum is

$$
\begin{gathered}
\mathrm{H}_{\mathrm{C}}(\mathrm{k} ; \mathrm{t}) \\
=\mathrm{hu}_{\mathrm{i}}(\mathrm{k} ; \mathrm{t}) \mathrm{b}(\mathrm{k} ; \mathrm{t})^{\mathrm{a}}+\mathrm{u}_{\mathrm{i}}(\mathrm{k} ; \mathrm{t})^{\mathrm{a}} \mathrm{b}(\mathrm{k} ; \mathrm{t}) \mathrm{i}
\end{gathered}
$$

and the triadic interaction function of cross helicity is

$$
\begin{gathered}
T_{H c}(k ; p ; q)={ }^{i} M_{i j k}(k) b_{i}(k ; t)^{a} \\
f\left[u_{j}(p ; t) u_{k}(q ; t) i b(p ; t) b_{k}(q ; t)\right] \AA \\
+M_{i j k}^{B}(k)^{a} u_{i}(k ; t) b(p ; t)^{a} u_{k}(q ; t)^{a}+c: c:
\end{gathered}
$$

\section{EDQNM Closure Equations}

The EDQNM equations for the kinetic energy and helicity spectra, $E(k ; t)$ and $E^{H}(k ; t)$, and magnetic energy and helicity spectra, $E_{B}(k ; t)$ and $E_{B}^{H}(k ; t)$ are an excellent means for obtaining the detailed triadic transfer functions for both total energy and cross helicity. An illustrative calculation of the velocity triadic transfer spectrum is shown in Fig. 1. In the full paper, detailed analysis of all transfer spectra and interacting scales will be presented to elucidate the fundamental dynamics of M HD turbulence.

In the three-dimensional case, the energy spectra evolution equations are ${ }^{11}$ :

$$
\begin{aligned}
& \mu \frac{@}{\mathrm{a} t}+2 \stackrel{\circ k^{2}}{\mathrm{q}} \mathrm{E}(\mathrm{k} ; \mathrm{t}) \\
& ={ }_{\Phi}^{Z Z} \mu_{k p q}^{3} T^{v v}+T^{£}+T^{v B}+T^{B B}+T^{\prime} \\
& \text { f dpdq; } \\
& \mu \frac{@}{@}+2^{3} k^{2} E_{B}(k ; t)
\end{aligned}
$$

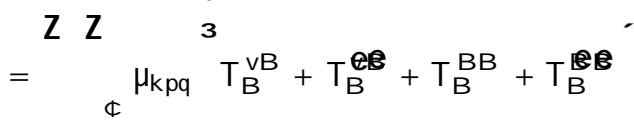

$$
\begin{aligned}
& \mu \quad \mathrm{fdpdq} \text {; }
\end{aligned}
$$

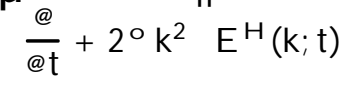

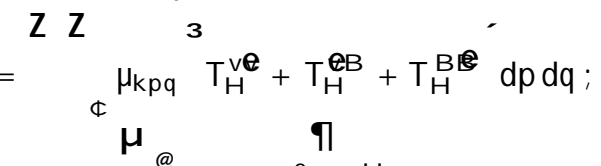

$$
\begin{aligned}
& \stackrel{\frac{@}{\circledast}}{\infty}+2^{3} k^{2} E_{B}^{H}(k ; t)
\end{aligned}
$$

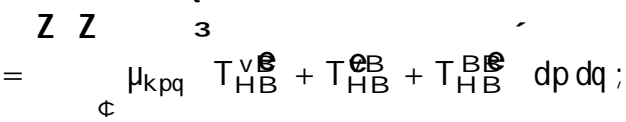

where

$$
\begin{aligned}
& T^{v v}(k ; p ; q ; t)=\frac{k}{p q} q_{k p q}{ }^{f}{ }^{2} E(p ; t) ; p^{2} E(k ; t) \\
& f E(q ; t) \text {; } \\
& T^{\circledast ; p ; q ; t)}=i \frac{1}{p q^{2}} c_{k p q} \\
& f \stackrel{f}{k^{2}} E^{H}(p ; t) ; p^{2} E^{H}(k ; t){ }^{\infty} E^{H}(q ; t) \text {; }
\end{aligned}
$$




$$
\begin{aligned}
& T^{v B}(k ; p ; q ; t)=\frac{k p}{q} c_{k p q} E(k ; t) E_{B}(q ; t) ; \\
& T^{B} B(k ; p ; q ; t)=\frac{k^{3}}{p q} c_{k p q} E_{B}(p ; t) E_{B}(q ; t) \text {; } \\
& T^{\mathbb{E}}(k ; p ; q ; t)=i \frac{k^{3}}{2 p} h_{k p q} E_{B}^{H}(p ; t) E_{B}^{H}(q ; t) ; \\
& T_{B}^{v B}(k ; p ; q ; t)=\frac{k^{5}}{p^{3} q} c_{k p q} E(p ; t) E_{B}(q ; t) \\
& +\frac{k}{p q} h_{k p q}{ }^{f} k^{2} E_{B}(p ; t) ; p^{2} E_{B}(k ; t){ }^{\alpha} E(q ; t) \text {; } \\
& T_{B}^{E}(k ; p ; q ; t)=\frac{k q}{p} h_{k p q} \frac{k^{2}}{p^{2}} E^{H}(p ; t) E_{B}^{H}(q ; t) \\
& i \frac{p^{2}}{q^{2}} E^{H}(q ; t) E_{B}^{H}(k ; t) ; \\
& \mathrm{T}_{\mathrm{B}}^{\mathrm{BB}}(\mathrm{k} ; \mathrm{p} ; \mathrm{q} ; \mathrm{t})=\mathrm{i} \frac{\mathrm{k}^{3}}{\mathrm{pq}} \mathrm{c}_{k p q} \mathrm{E}_{\mathrm{B}}(\mathrm{k} ; \mathrm{t}) \mathrm{E}_{\mathrm{B}}(\mathrm{q} ; \mathrm{t}) ; \\
& T_{B}^{B}(k ; p ; q ; t)=k p^{2} e_{k p q} E_{B}^{H}(k ; t) E_{B}^{H}(q ; t) ; \\
& T_{H}^{v e}(k ; p ; q ; t)=\frac{k}{p q} b_{p q q} k^{f} E^{H}(p ; t) i p^{2} E^{H}(k ; t){ }^{\alpha} \\
& f E(q ; t) \\
& i \frac{k^{2} p}{q^{2}} c_{k p q}[E(p ; t) ; E(k ; t)] E^{H}(q ; t) \text {; } \\
& T_{H}^{\otimes B}(k ; p ; q ; t)=i \frac{k p}{q} c_{k p q} E^{H}(k ; t) E_{B}(q ; t) ; \\
& \left.T_{H}^{B} \mathbb{E}_{(k ; p ; q} t\right)=\frac{k^{4}}{q} f_{k p q} E_{B}^{H}(p ; t) E_{B}(q ; t) ; \\
& T_{H B}^{v E R}(k ; p ; q ; t)=\frac{k}{p q} h_{k p q} \stackrel{f}{k^{2}} E_{B}^{H}(p ; t) i p^{2} E_{B}^{H}(k ; t) \\
& f E(q ; t) \\
& T_{H B}^{\oplus B}(k ; p ; q ; t)=\frac{k}{p q} h_{k p q} \frac{k^{2}}{p^{2}} E^{H}(p ; t) E_{B}(q ; t) \\
& i \frac{p^{2}}{k^{2}} E^{H}(q ; t) E_{B}(k ; t) \text {; }
\end{aligned}
$$

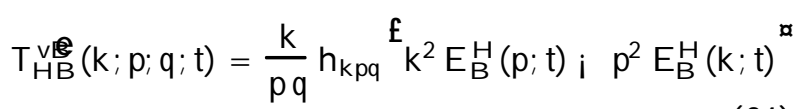

$$
\begin{gathered}
f E(q ; t) ; \\
T_{H B}^{E B}(k ; p ; q ; t)=\frac{k}{p q} h_{k p q} \frac{k^{2}}{p^{2}} E^{H}(p ; t) E_{B}(q ; t) \\
i \frac{p^{2}}{k^{2}} E^{H}(q ; t) E_{B}(k ; t) ; \\
T_{H B}^{B E}(k ; p ; q ; t)=\frac{p^{2}}{k} e_{k p q} E_{B}(k ; t) E_{B}^{H}(q ; t) \\
i \frac{k p}{q} j_{k p q} E_{B}(q ; t) E_{B}^{H}(k ; t) ;
\end{gathered}
$$

$(x, y$, and $z$ are the cosines of the interior angles opposite the sides formed by $k, p$, and $q$, respectively) and the three-point eddy correlation timescale is

$$
\begin{aligned}
& \mu_{k p q}(t)=\frac{1 j \exp \left[i^{1}{ }_{k p q}(t) t\right]}{{ }^{1}{ }_{k p q}(t)} ; \\
& { }^{1}{ }_{k p q}(t)={ }^{1} k(t)+{ }^{1}{ }_{p}(t)+{ }^{1}{ }_{q}(t)
\end{aligned}
$$

with eddy damping rate

$$
\begin{gathered}
{ }_{1}{ }_{k}(t)=\left(0+{ }^{3}\right) k^{2} \\
+0: 177 K o^{3=2}{ }^{" Z_{k}} p^{2}\left[E(p ; t)+E_{B}(p ; t)\right] d p \\
+\frac{2}{3} k_{0}{ }_{0} Z_{k} E_{B}(p ; t) d p
\end{gathered}
$$

where $\mathrm{Ko}$ is the assumed Kolm ogorov constant. 


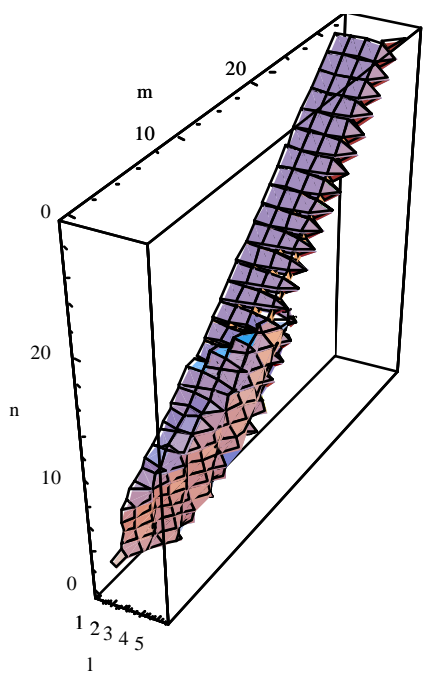

Fig. 1 Three-dimensional contour plot of the triadic kinetic energy transfer function [the sum of (20) \{(24)] obtained from the EDQNM model; the integers I, $m$, and $n$ corresp ond to wavenumbers $k, p$, and $q$.

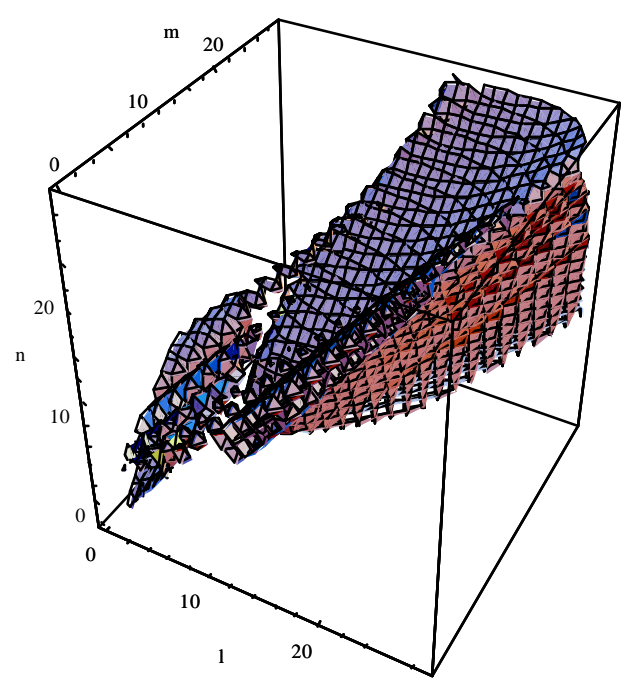

Fig. 2 Three-dimensional contour plot of the triadic magnetic energy transfer function [the sum of (25) \{(28)] obtained from the EDQNM model; the integers $\mathrm{l}, \mathrm{m}$, and $\mathrm{n}$ corresp ond to wavenumbers $k, p$, and $q$.

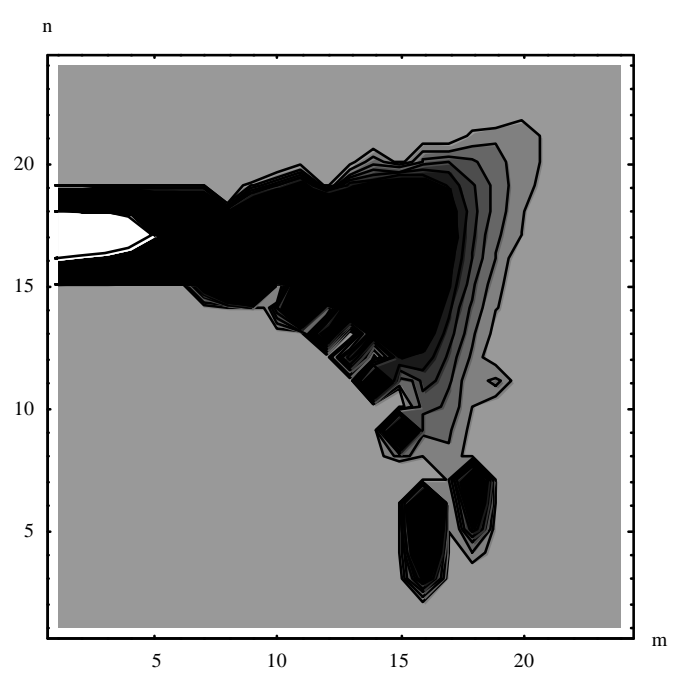

Fig. 3 Two-dimensional contour plot of the triadic kinetic energy transfer function [the sum of (20) $\{(24)]$ obtained from the EDQNM model for mode $\mathrm{I}=17$; the integers $\mathrm{m}$ and $\mathrm{n}$ correspond to wavenumbers $\mathrm{p}$ and $\mathrm{q}$.

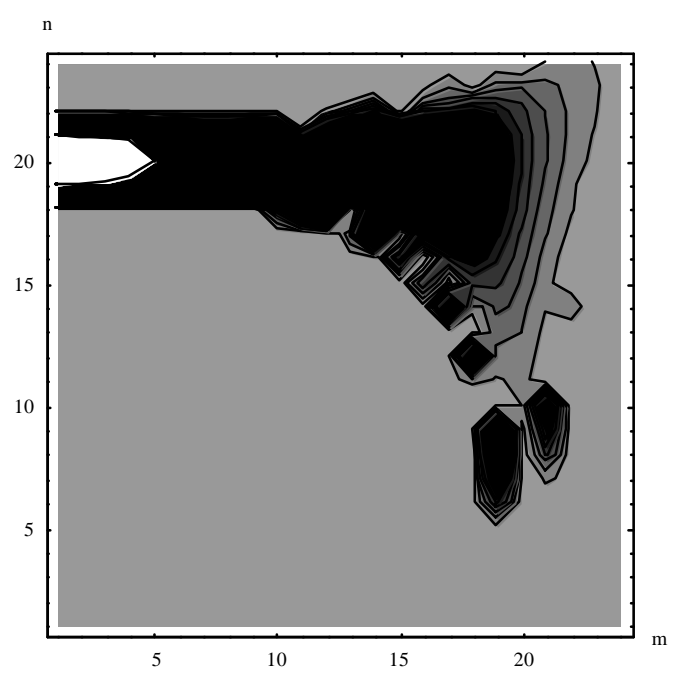

Fig. 4 Two-dimensional contour plot of the triadic kinetic energy transfer function [the sum of (20) $\{(24)]$ obtained from the EDQNM model for mode $\mathrm{I}=20$; the integers $\mathrm{m}$ and $\mathrm{n}$ correspond to wavenumbers $\mathrm{p}$ and $\mathrm{q}$. 


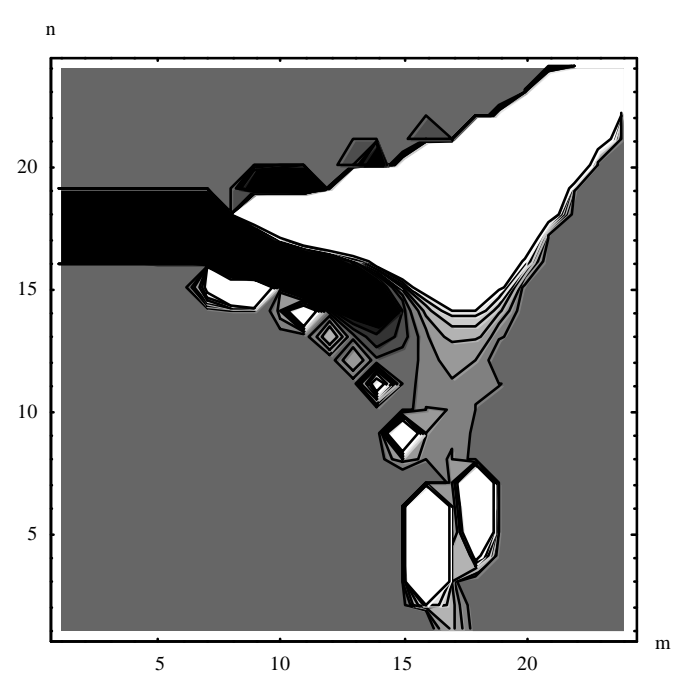

Fig. 5 Two-dimensional contour plot of the triadic magnetic energy transfer function [the sum of (25) $\{(28)]$ obtained from the EDQNM model for mode I = 17; the integers $\mathrm{m}$ and $\mathrm{n}$ correspond to the wavenumbers $p$ and $q$

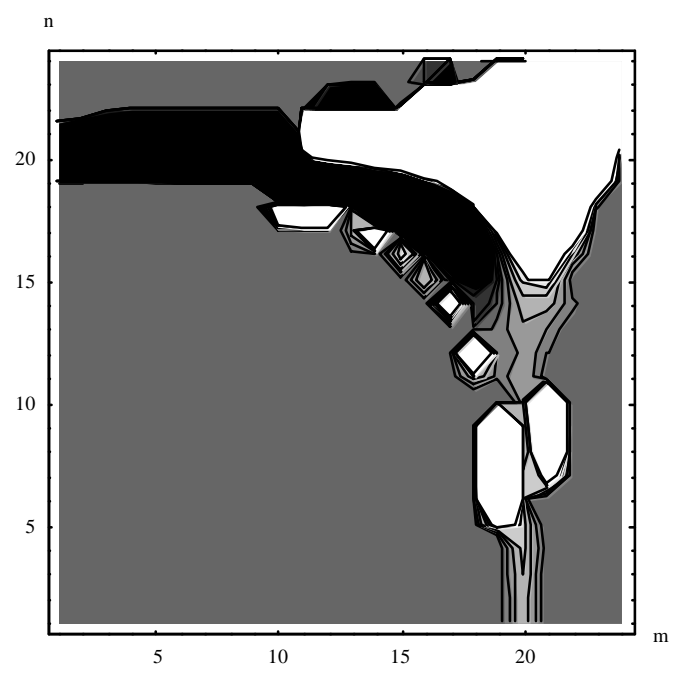

Fig. 6 Two-dimensional contour plot of the triadic magnetic energy transfer function [the sum of $(25)\{(28)$ ] obtained from the EDQNM model for mode I = 20; the integers $\mathrm{m}$ and $\mathrm{n}$ correspond to the wavenumbers $p$ and $q$

\section{Results and Conclusions}

The triadic transfer functions $(20)\{(36)$ were computed for assumed $\mathrm{K}$ olmogorov inertial subrange energy spectra

$$
\begin{aligned}
& E(k)=E^{H}(k) \\
& =K o^{22=3} k^{i} 5=3 \\
& E_{B}(k)=E_{B}^{H}(k) \\
& =K o_{B}{ }_{B}^{2=3} k^{i} 5=3
\end{aligned}
$$

with $\mathrm{KO}=\mathrm{KO}_{\mathrm{B}}=1: 7$ and $2={ }^{2}=1$. The wavenumber range was k; p; 2 [1;53:81], with the discrete wavenumbers given by

$$
k_{1}=k_{\min } 2^{(1 ; 1)=4}
$$

with $\mathrm{k}_{\min }=1: 0$ and $\mathrm{I}=1 ;::: ; 24$.

The EDQNM model is a useful tool for providing insight into the energy transfer processes in three-dimensional, isotropic MHD turbulence. The present study considered only the energy transfer, and a study of the helicity transfers will be undertaken in the future. Additionally, the subject of subsequent research will be the study of the locality of the energy transfer and the dynamics of interacting scales. It is expected that the results of these studies will have important implications for space and astrophysical plasmas.

\section{Acknowledgements}

This work was performed under the auspices of the U.S. Department of Energy by the U niversity of California Lawrence Livermore National Laboratory under contract No. W-7405-E ng-48. This research was also partly supported by the National Science Foundation under grant ATM 9814045. The calculations were performed using Mathematica ${ }^{R}$ 4.1.

\section{References}

1. D. Fyfe, D. Montgomery, G. J oyce, J. Plasma Phys., 17, 369 ( 1977)

2. R. H. Kraichnan, Phys. Fluids, 8, 1385 (1965)

3. L. Woltjer, Proc. Nat. Acad. Sci., 44, 489 (1958)

4. U. Frisch, A. Pouquet, J. Løorat, and A. Mazure, J. Fluid M ech., 68, 769 (1975) 
5. Y. Zhou and W. H. M atthaeus, J. Geophys. Res., 95, 10291 (1990)

6. Y. Zhou and W. H. M atthaeus, J. Geophys. Res., 95, 14881 (1990)

7. Y. Zhou and W. H. M atthaeus, J. Geophys. Res., 95, 10310 (1990)

8. Y. Zhou, Phys. Fluids A, 5, 1092 ( 1993)

9. Y. Zhou, Phys. Fluids A, 5, 2511 (1993)

10. Y. Zhou, P. K. Y eung, and J . G. Brasseur, P hys. Rev. E, 53, 1261 (1996)

11. A. Pouquet, U. Frisch, and J. Lorat, J. Fluid Mech., 77, 321 (1976) 